Published in final edited form as:

Anal Chem. 2019 October 01; 91(19): 12167-12172. doi:10.1021/acs.analchem.9b03162.

\title{
mTMT: An Alternative, Nonisobaric, Tandem Mass Tag Allowing for Precursor-Based Quantification
}

\author{
Joao A. Paulo*, Steven P. Gygi \\ Department of Cell Biology, Harvard Medical School, Boston, Massachusetts 02115, United \\ States
}

\begin{abstract}
Stable isotope labeling of peptides is the basis for numerous mass-spectrometry-based quantification strategies. Isobaric tagging and metabolic labeling, namely, tandem mass tagging (TMT) and SILAC, are among the most widely used techniques for relative protein quantification. Here we report an alternative, precursor-based quantification method using nonisobaric TMT variants: TMTzero (TMT0) and superheavy TMT (shTMT). We term this strategy mass difference tandem mass tagging (mTMT). These TMT variants differ by 11 mass units; however, peptides labeled with these reagents coelute, analogous to SILAC-labeled peptide pairs. As a proof-ofconcept, we profiled the proteomes of two cell lines that are frequently used in neuroscience studies, SH-SY5Y and SVGp12, using mTMT and standard SILAC-labeling approaches. We show similar quantified proteins and peptides for each method, with highly correlated fold-changes between workflows. We conclude that mTMT is a suitable alternative for precursor-based protein quantification.
\end{abstract}

\section{Graphical Abstract}

\footnotetext{
*Corresponding Author: joao_paulo@hms.harvard.edu.

Supporting Information

The Supporting Information is available free of charge on the ACS Publications website at DOI: 10.1021/acs.anal-chem.9b03162. Supplemental methods, Venn diagrams illustrating quantified peptide overlap between replicates and workflows, and protein classification using gProfiler (PDF)

Proteins identified in the data set (XLSX)

Peptides identified in the data set (PDF)

The authors declare no competing financial interest.
} 
TMTO<smiles>CC1CCCC(C)N1CC(=O)NCCC(=O)ON1C(=O)CCC1=O</smiles>

ShTMT

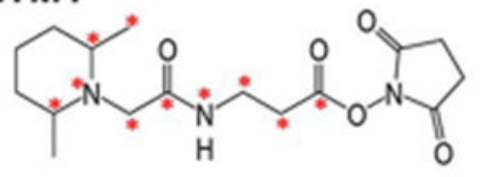

ShTMT

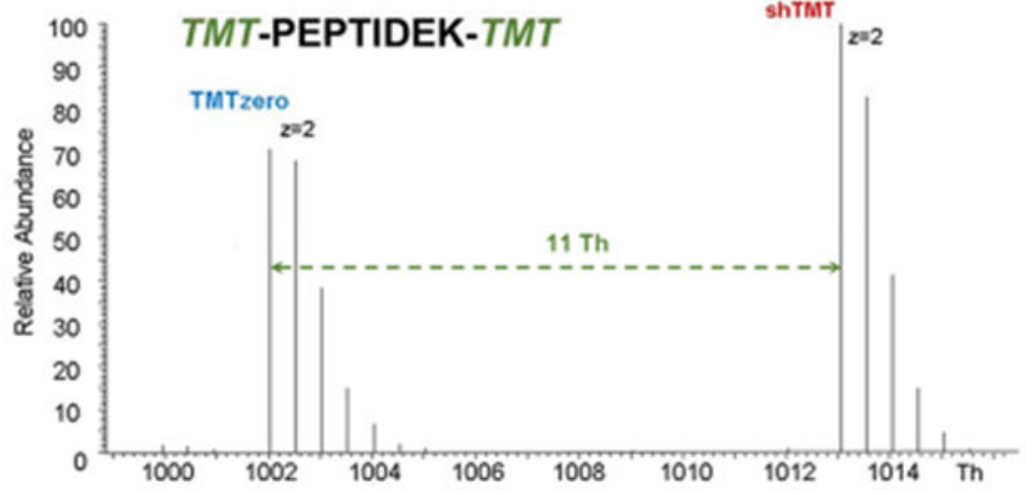

Developments in quantitative proteomics have yielded many innovative and streamlined strategies for comprehensive proteome profiling. Stable isotope labeling in cell culture (SILAC) has been used for mass-spectrometry-based proteome profiling for over a decade. 1,2 Metabolic labeling enables the near complete incorporation of stable-isotope-labeled lysine or arginine in proteins from cell-culture-based systems. Metabolic labeling requires heavy-isotope-labeled amino acids, in addition to extended time for full label incorporation, which may be inefficient for slow-dividing cell lines. Moreover, full heavy isotope labeling is more involved for higher organisms, such as mice, ${ }^{3}$ and infeasible for humans. Reductive dimethylation remains an attractive, inexpensive option that evades these caveats, but its use is dampened by slightly more complicated sample processing, compounded by potential deuterium-based retention time differences. ${ }^{4,5}$ Alternatively, isobaric labeling with tandem mass tags (TMT) is rapidly emerging as the method of choice for multiplexed relative proteome quantification. ${ }^{6}$ These NHS-ester reactive reagents incorporate stable-isotopelabeled residues that are distributed between the reporter and balancer groups to ensure that the different tags have equal nominal masses. ${ }^{6}$ Upon fragmentation, the intensity of the reporter ions represents the relative quantification of the precursor peptide across the channels used. The TMT labeling procedure has been optimized for efficiency and robustness. ${ }^{7,8}$

Although multiplexing isobaric reagents allows for profiling of over 10 samples in a single experiment, such scale is often unnecessary when sample numbers are limited or simple binary investigations are desired, as is often the impetus for SILAC experiments. As such, we developed an alternative to SILAC labeling using two derivatives of the TMT molecule, specifically, TMTzero (TMT0) and superheavy TMT (shTMT). We term this workflow mass difference tandem mass tagging (mTMT), reflecting its similarity to mTRAQ. ${ }^{9,10}$ Although structurally identical, these two mTMT reagents differ in the number of stable isotope labels ( 0 for TMT0 and 11 for shTMT) and thereby are not isobaric, yet they coelute when separated via reversed-phase chromatography. Two additional nonisobaric TMT labels are 
available: TMT6-11 which is 5 and 6 Th away from TMT0 and shTMT, respectively, and TMTduplex, which is 1 and 10 Th away, respectively. These labels were not used here because of the potential of isotopic envelope overlap and for experimental simplicity. As with SILAC, mTMT quantification is precursor-based. ${ }^{1,2}$ The general concept of nonisobaric labeling presented here is not limited to TMT, as any succinimidyl ester molecule having the acyl portion or a similar reagent that is labeled with heavy isotopes would be effective. We have used mTMT previously to develop an internal standard for assessing recovery in phosphoproteomic experiments using precursor-based quantification. ${ }^{11}$ However, here we have expanded the concept to profile comprehensively the proteomes of two cell lines, using SILAC as our benchmark for comparison.

To showcase mTMT, we compared two human cell lines that are often used in neuroscience research, specifically, SH-SY5Y and SVGp12. SH-SY5Y is a subclone of SK-N-SH, which was isolated from bone marrow. ${ }^{12,13}$ SH-SY5Y has been used for decades as an in vitro model of neuronal function, differentiation, and dysregulation. Studies using this cell line include those focusing on neurogenesis, as well as neurodegeneration research related to Parkinson's and Alzheimer's diseases. ${ }^{14-17}$ Less commonly used yet nonetheless valuable in neurobiology research, SVGp12 was established by transfecting cultured human astroglia cells of brain origin with SV40-devived DNA. ${ }^{18}$ SVGp12 has been used in studies involving tumor formation, proliferation, and apoptosis related to glioma. ${ }^{19-21}$ Here we perform the first comparative proteome profiling of these two cell lines using our mTMT labeling strategy. Our data highlight the similarities in quantification when using either mTMT or the conventional SILAC strategy. We thereby demonstrate that the mTMT strategy, which does not require metabolic labeling, is well-suited for mass-spectrometry-based quantitative proteomic analyses whenever binary comparisons are sufficient.

\section{METHODS}

Materials.

TMT0 (No. 90067) and shTMT (No. A43073) were from ThermoFisher Scientific (Rockford, IL). Dulbecco's modified Eagle's medium (DMEM) and fetal bovine serum (FBS) were from LifeTechnologies (Waltham, MA). SILAC Protein Quantitation Kit (Trypsin) - DMEM and Trypsin was purchased from ThermoFisher Scientific. Lys-C was bought from Fujifilm Wako (Richmond, VA). Water and organic solvents were from J.T. Baker (Center Valley, PA). The SVGp12 (C859) and SH-SY5Y cell lines were purchased from ATCC (Manassas, VA). Unless otherwise noted, all other chemicals were from ThermoFisher Scientific.

\section{Sample Preparation.}

Details on cell growth and harvesting, cell lysis and protein digestion, tandem mass tag labeling, off-line basic pH reversed-phase (BPRP) fractionation, and LC-MS/MS analysis can be found in the Supplemental Methods. 


\section{Data Analysis.}

Raw MS data files were processed using MaxQuant (v. 1.6.2.10) with the integrated search engine Andromeda ${ }^{22}$ using default settings for SILAC-based quantification. MS/MS spectra were searched against the UniProt human proteome sequence database (UP000006640_9606.fasta). For mTMT experiments, the labels were set as follows: shTMT (235.176 $74 \mathrm{Th})$ on K and N termini were "heavy" labels, whereas TMT0 (224.152 $48 \mathrm{Th})$ on $\mathrm{K}$ and $\mathrm{N}$ termini were "light" labels. For SILAC experiments, heavy labeled samples contained Lys8 (+8.014 $20 \mathrm{Th})$ and Arg10 (+10.008 $27 \mathrm{Th})$, whereas "light" samples were unmodified. For protein-level comparisons, peptides were identified, quantified, and collapsed to a $1 \%$ peptide false discovery rate (FDR) and then collapsed further to a final protein-level FDR of $1 \%$, which resulted in a final peptide level FDR of $<0.1 \%$. RAW files have been deposited to the ProteomeXchange Consortium via the PRIDE ${ }^{23}$ partner repository with the data set identifier PXD014545 (username: reviewer00843@ebi.ac.uk, password: CUdEeF7U).

\section{RESULTS AND DISCUSSION}

\section{mTMT Workflow Simplified Sample Preparation by Precluding the Need for Metabolic Labeling.}

We developed the mTMT workflow to enable precursor-based proteome quantification without the need for metabolic labeling. As a proof-of-concept, we compared mTMT to the most widely used precursor-based quantitative proteomics method, SILAC. We showcased the similarities between these workflows by comparing the proteomes of two cell lines, namely, SVGp12 and SH-SY5Y (Figure 1).

A key advantage of mTMT is that it facilitates the labeling of any sample in a single step without requiring metabolically encoded heavy isotopes. For human tissues, metabolic labeling is typically not feasible and other methods of quantification (such as label-free quantification, data independent acquisition, and isobaric tagging) may be required. Another advantage of mTMT over metabolic labeling is that no additional time is needed to maintain and actively culture the cells to allow for the incorporation of stable-isotope-labeled amino acids. For example, here, both cell lines underwent 10 passages to ensure complete heavy isotope labeling. SH-SY5Y was particularly challenging because of its relatively long doubling time. Much like SILAC labeling, efficient mTMT labeling is crucial for accurate quantification. As such, prior to mixing the mTMT reagents, we tested labeling efficiency by analyzing $2 \%$ of each mTMT-labeled sample and searching TMT as a variable modification as performed routinely with TMT6/10/11-plex analysis. ${ }^{8}$ Our labeling efficiency was $>99 \%$ for the unfractionated mTMT samples.

The cost of mTMT is also less. Specifically, to label $50 \mu \mathrm{g}$ of peptide requires $100 \mu \mathrm{g}$ of each TMT reagent, which currently costs $\sim \$ 5$ for TMT0 and $\sim 25$ for shTMT. This cost is minimal compared with that of stable-isotope-labeled amino acids plus the time and associated reagents needed for full heavy label incorporation, yet mTMT sample preparation remains more expensive than label-free techniques. However, when only examining two samples, mTMT retains the advantage that both conditions are analyzed and processed 
together after labeling. This notion is important for achieving deeper proteome coverage, such as with off-line orthogonal fractionation (as performed here). Multiplexed analysis reduces the risk of missing values resulting from chromatographic differences when conditions are analyzed together. Nonetheless, metabolic labeling does maintain an advantage in that samples may be processed together earlier in the workflow, such as after protein precipitation, limiting potential technical variability due to separate processing of samples from different experimental conditions. Herein, the SILAC samples we mixed after cell lysis and before precipitation. We have observed that precipitation, particularly chloroform-methanol and TCA precipitation, are prone to sample loss due to human error during wash aspirations, thereby prompting us to avoid separate sample precipitation whenever possible.

\section{Label-Reversed Replicate Analyses of mTMT Reveal Overlaps in Protein Identification Similar to Those Observed in a Set of Replicate Samples Using SILAC.}

Our analysis for each workflow was performed in duplicate. As is common for SILAC sample processing, ${ }^{1,2}$ we reversed the labels of the cell lines for each replicate. That is, in the first replicate, SH-SY5Y was labeled in light media for SILAC and with TMT0 for mTMT, whereas in the second replicate, SH-SY5Y was labeled with heavy SILAC media and shTMT, and SVGp12 had the corresponding label in each pair. The proteins were inferred from approximately 60000 peptides per replicate in each data set (Table 1). On average, $\sim 8500$ proteins were quantified with mTMT, whereas $\sim 7600$ were quantified with SILAC. This difference may be due to the metabolism of the stable-isotope-labeled amino acids (e.g., conversion of arginine to proline), but further investigation is needed. ${ }^{24}$ Lists of proteins and peptides, along with associated metrics, are available in Supplemental Tables S1 and S2, respectively. Within each workflow, the overlap between replicates was high ( $80 \%$ for SILAC and $85 \%$ for TMT) (Figure 2A,B). Comparing the intersection of the two sets of replicates revealed 6382 proteins that were quantified in each data set, which corresponded to over $77 \%$ of proteins being quantified in both (Figure 2C). Likewise, we investigated the peptide overlap on the basis of amino acid sequences only (i.e., posttranslational modifications and tags were stripped from the peptides). Our data revealed that over 31000 peptides (36\%) were common between the SILAC replicates (Supplemental Figure 1A), and over 36000 peptides (40\%) were common between mTMT replicates. We then compared the total peptides quantified in each workflow (Supplemental Figure 1B). We expected the overlap to be low as a result of disparities in sample preparation and the fundamentally different labeling strategies, which could result in characteristically distinct sets of peptides. However, the peptide overlap between the workflows closely resembled that between replicates. The two data sets exhibited an overlap of over 48000 peptides, corresponding to over 36\% (Supplemental Figure 1C), a value that was close to that of the label-reversed replicates discussed above.

\section{Protein Expression Profiles are Highly Correlated within mTMT and SILAC Replicates and across Workflows.}

We plotted the distribution of the protein level fold-changes ( $\log _{2}$ (SH-SH5Y/SVGp12)) for each of the four data sets (Figure 2D). We observed that the fold-change distributions among all data sets were nearly indistinguishable whether processed via mTMT or SILAC. For a 
more quantitative assessment among data sets, we constructed a correlation plot matrix using all four data sets (Figure 2E). Each data point represented the $\log _{2}$ ratio of the relative protein abundance between the two cell lines (i.e., $\log _{2}(\mathrm{SH}-\mathrm{SH} 5 \mathrm{Y} / \mathrm{SVG} 12)$ ). From here, we calculated the Pearson correlation coefficients $(r)$ between pairs of data sets using proteins that are in common among all four data sets $(n=6382)$. As expected, label-reversed replicates demonstrated stronger correlation values, approaching Pearson coefficients of 0.9. The correlation coefficient between the mTMT and SILAC data sets were slightly lower at $0.83-0.84$. Moreover, the cross-workflow comparisons confirmed that the data agreed regardless of workflow. We also calculated the coefficient of variation over all redundant quantifiable peptides for a given protein. SILAC quantification resulted in a median error of $21 \%$, and that of mTMT was slightly higher at $26 \%$, which was close to what may be expected. ${ }^{25,26}$ Although not performed here, the dynamic range for mTMT compared with that of SILAC should also be determined. ${ }^{26}$ Nonetheless, the similarity between protein quantification strategies provided further support for mTMT as an alternative workflow for precursor-based quantification.

\section{mTMT and SILAC Workflows Highlight Common Protein Abundance Differences across SVGp12 and SH-SY5Y Cell Lines.}

After illustrating the similarities in global fold-change distributions and determining the correlations of these protein abundance alterations between mTMT and SILAC, we focused on the types of proteins that were highly different between cell lines. We plotted the $\log _{2}$ ratio of protein abundance between the SH-SY5Y and SVGp12 cell lines for SILAC (Figure $3 \mathrm{~A}$ ) and mTMT (Figure 3B). We ranked all proteins in each data set by increasing foldchange. A similar pattern was obtained from both workflows and comparable numbers of differentially expressed proteins ( \pm 2 -fold difference) were also observed (Table 1 ), which was consistent with the data discussed above. As we had only two measurements per comparison group, we were unable to properly perform significance testing of these foldchanges. We chose a fold-change threshold of \pm 2 as it approximated two standard deviations in fold-change. We emphasize that overall the quantitative data correlated well (Figure 2E); however, the significance of individual fold-changes would benefit from a third or more replicates and permit testing statistical significance and proper multiple testing correction ${ }^{27}$.

Next, we used gProfiler to extract the enriched molecular functions of all significantly altered proteins. ${ }^{28}$ As expected, the mTMT and SILAC workflows resulted in similar enriched functions for the altered proteins. We listed the highest scoring gProfiler categories in Supplemental Table S3. Proteins of higher abundance in SVGp12 cells included those associated with focal adhesion, ECM-receptor interaction, actin cytoskeleton regulation, and proteoglycans in cancer. In particular, enrichment in the categories of focal adhesion and ECM-receptor interaction were anticipated as glial cells are frequently associated with the regulation of extracellular matrix. ${ }^{29}$ We highlighted 10 proteins that were among the 50 most abundant in SVGp12 cells compared with in SH-SY5Y cells and which were present in both SILAC and mTMT data sets (Figure 3A,B, left). The proteins were listed in order of lowest to highest fold-change and included moesin (MSN), nexilin (NEXN), and coactosin-like protein (COTL1), which are involved in cytoskeletal restructuring, whereas PTPN14 is a tyrosine-protein phosphatase that has a role in cell migration and growth. This result was 
expected as the morphologies of these two cell lines were very different, with SVGp12 having a round shape and SH-SY5Y having neuron-like projections (neurites).

In contrast, proteins of higher abundance in SH-SY5Y cells included those associated with neurons, in particular, axons and neuron projections. Again, we highlighted 10 proteins that were among the 50 most abundant in SH-SY5Y cells compared with in SVGp12 cells (Figure 3A,B, right). The proteins, listed in order of lowest to highest fold-change, included nestin (NES), which is required for survival, renewal, and mitogen-stimulated proliferation of neural progenitor cells; dihydropyrimidinase-related protein 4 (DPYSL4), which plays a role in axon guidance and neuronal growth cones; disintegrin- and metalloproteinasedomain-containing protein 22 (ADAM22), which is a probable ligand for integrin in the brain; and 4-aminobutyrate aminotransferase (ABAT), which catalyzes the conversion of $\gamma$ aminobutyrate and L- $\beta$-aminoisobutyrate to succinate semialdehyde. As SH-SY5Y cells are considered cell line surrogates for neurons in neurotransmitter studies, we expected enrichment in these categories. ${ }^{30}$ These data showed that the baseline proteomes differed between these cell lines. As such, future experiments may investigate how certain perturbations affect the proteomes of these cell lines.

\section{CONCLUSIONS}

We developed the mTMT workflow to permit precursor-based quantification regardless of sample origin. We compared our data from the mTMT workflow to that from a traditional SILAC strategy. Similar results were obtained with respect to the number of proteins quantified, relative protein abundances among cell lines, and molecular functions ofstatistically different proteins. Although isobaric tagging has the advantage of higher order multiplexing, instruments capable of obtaining MS3 spectra are often required to prevent ion interference induced ratio compression. ${ }^{31,32}$ mTMT avoids this caveat through MS1-based quantification, which can be performed on a wide range of mass spectrometers, such as a $\mathrm{Q}$ Exactive. Our data support that the mTMT workflow is a well-suited alternative to SILAC, with the advantages of having no limitation on sample type and not requiring an extended time period for metabolic incorporation of stable isotopes.

\section{Supplementary Material}

Refer to Web version on PubMed Central for supplementary material.

\section{ACKNOWLEDGMENTS}

We would like to thank members of the Gygi Lab at Harvard Medical School. This work was funded in part by NIH grants GM132129 (J.A.P.) and GM67945 (S.P.G.).

\section{REFERENCES}

(1). Oda Y; Huang K; Cross FR; Cowburn D; Chait BT Proc. Natl. Acad. Sci. U. S. A 1999, 96 (12), 6591-6. [PubMed: 10359756]

(2). Ong SE; Blagoev B; Kratchmarova I; Kristensen DB; Steen H; Pandey A; Mann M Mol. Cell. Proteomics 2002, 1 (5), 376-86. [PubMed: 12118079] 
(3). Kruger M; Moser M; Ussar S; Thievessen I; Luber CA; Forner F; Schmidt S; Zanivan S; Fassler R; Mann M Cell 2008, 134 (2), 353-64. [PubMed: 18662549]

(4). Tolonen AC; Haas WJ Visualized Exp. 2014, No. 89, e51416.

(5). Boersema PJ; Raijmakers R; Lemeer S; Mohammed S; Heck AJR. Nat. Protoc 2009, 4, 484. [PubMed: 19300442]

(6). Thompson A; Schaefer J; Kuhn K; Kienle S; Schwarz J; Schmidt G; Neumann T; Johnstone RAW; Mohammed AKA; Hamon C Anal. Chem 2003, 75 (8), 1895-904. [PubMed: 12713048]

(7). Zecha J; Satpathy S; Kanashova T; Avanessian SC; Kane MH; Clauser KR; Mertins P; Carr SA; Kuster B Mol. Cell. Proteomics 2019, 18, 1468. [PubMed: 30967486]

(8). Navarrete-Perea J; Yu Q; Gygi SP; Paulo JA J. Proteome Res. 2018, 17 (6), 2226-2236. [PubMed: 29734811]

(9). DeSouza LV; Taylor AM; Li W; Minkoff MS; Romaschin AD; Colgan TJ; Siu KW J. Proteome Res. 2008, 7 (8), 3525-34. [PubMed: 18630974]

(10). Oppermann FS; Klammer M; Bobe C; Cox J; Schaab C; Tebbe A; Daub H J. Proteome Res. 2013, 12 (9), 4089-100. [PubMed: 23898821]

(11). Paulo JA; Navarrete-Perea J; Erickson AR; Knott J; Gygi SP J. Am. Soc. Mass Spectrom. 2018, 29 (7), 1505-1511. [PubMed: 29671274]

(12). Biedler JL; Helson L; Spengler BA Cancer Res. 1973, 33 (11), 2643-2652. [PubMed: 4748425]

(13). Biedler JL; Roffler-Tarlov S; Schachner M; Freedman LS Cancer Res. 1978, 38 (11), 3751-3757. [PubMed: 29704]

(14). Jeong HJ; Kim DW; Woo SJ; Kim HR; Kim SM; Jo HS; Park M; Kim DS; Kwon OS; Hwang IK; Han KH; Park J; Eum WS; Choi SY Mol. Cells 2012, 33 (5), 471-8. [PubMed: 22526393]

(15). Bartolome F; de la Cueva M; Pascual C; Antequera D; Fernandez T; Gil C; Martinez A; Carro E Alzheimer's Res. Ther. 2018, 10 (1), 24. [PubMed: 29458418]

(16). Shang Y; Liu M; Wang T; Wang L; He H; Zhong Y; Qian G; An J; Zhu T; Qiu X; Shang J; Chen Y Environ. Pollut 2019, 246, 763-771. [PubMed: 30623832]

(17). Zainal Abidin S; Fam SZ; Chong CE; Abdullah S; Cheah PS; Nordin N; Ling KH Gene 2019, 697, 201-212. [PubMed: 30769142]

(18). Henriksen S; Tylden GD; Dumoulin A; Sharma BN; Hirsch HH; Rinaldo CH J. Virol 2014, 88 (13), 7556-68. [PubMed: 24760884]

(19). Conde M; Michen S; Wiedemuth R; Klink B; Schrock E; Schackert G; Temme A BMC Cancer 2017, 17 (1), 889. [PubMed: 29282022]

(20). Cao L; Lei H; Chang MZ; Liu ZQ; Bie XH Biochem. Biophys. Res. Commun 2015, 462 (4), 389-95. [PubMed: 25982477]

(21). Gong F; Wang G; Ye J; Li T; Bai H; Wang W Oncol. Rep 2013, 30 (6), 2976-82. [PubMed: 24065186]

(22). Cox J; Neuhauser N; Michalski A; Scheltema RA; Olsen JV; Mann MJ Proteome Res. 2011, 10 (4), 1794-805.

(23). Perez-Riverol Y; Csordas A; Bai J; Bernal-Llinares M; Hewapathirana S; Kundu DJ; Inuganti A; Griss J; Mayer G; Eisenacher M; Perez E; Uszkoreit J; Pfeuffer J; Sachsenberg T; Yilmaz S; Tiwary S; Cox J; Audain E; Walzer M; Jarnuczak AF; Ternent T; Brazma A; Vizcaino JA Nucleic Acids Res. 2019, 47 (D1), D442-D450. [PubMed: 30395289]

(24). Ong SE; Kratchmarova I; Mann M J. Proteome Res. 2003, 2 (2), 173-81. [PubMed: 12716131]

(25). Carr SA; Abbatiello SE; Ackermann BL; Borchers C; Domon B; Deutsch EW; Grant RP; Hoofnagle AN; Huttenhain R; Koomen JM; Liebler DC; Liu T; MacLean B; Mani DR; Mansfield E; Neubert H; Paulovich AG; Reiter L; Vitek O; Aebersold R; Anderson L; Bethem R; Blonder J; Boja E; Botelho J; Boyne M; Bradshaw RA; Burlingame AL; Chan D; Keshishian H; Kuhn E; Kinsinger C; Lee JS; Lee SW; Moritz R; Oses-Prieto J; Rifai N; Ritchie J; Rodriguez H; Srinivas PR; Townsend RR; Van Eyk J; Whiteley G; Wiita A; Weintraub S Mol. Cell. Proteomics 2014, 13 (3), 907-17. [PubMed: 24443746]

(26). Lau HT; Suh HW; Golkowski M; Ong SE J. Proteome Res. 2014, 13 (9), 4164-74. [PubMed: 25077673] 
(27). Pascovici D; Handler DC; Wu JX; Haynes PA Proteomics 2016, 16 (18), 2448-53. [PubMed: 27461997]

(28). Reimand J; Arak T; Adler P; Kolberg L; Reisberg S; Peterson H; Vilo J Nucleic Acids Res. 2016, 44 (W1), W83-9. [PubMed: 27098042]

(29). Banerjee S; Bhat MA Annu. Rev. Neurosci 2007, 30, 235-58. [PubMed: 17506642]

(30). La Quaglia MP; Manchester KM J. Pediatr Surg 1996, 31 (2), 315-8. [PubMed: 8938368]

(31). Gygi JP; Yu Q; Navarrete-Perea J; Rad R; Gygi SP; Paulo JA J. Proteome Res. 2019, 18 (2), 687693. [PubMed: 30451507]

(32). Paulo JA; O’Connell JD; Gygi SP J. Am. Soc. Mass Spectrom. 2016, 27 (10), 1620-5. [PubMed: 27400695] 
A. тмто<smiles>CC1CCCC(C)N1CC(=O)NCCC(=O)ON1C(=O)CCC1=O</smiles>

ShTMT<smiles>CC1=CCC[C@@H](C)N1CC(=O)NCCC(=O)ON1C(=O)CCC1=O</smiles>

B.

SILAC

rep. 1

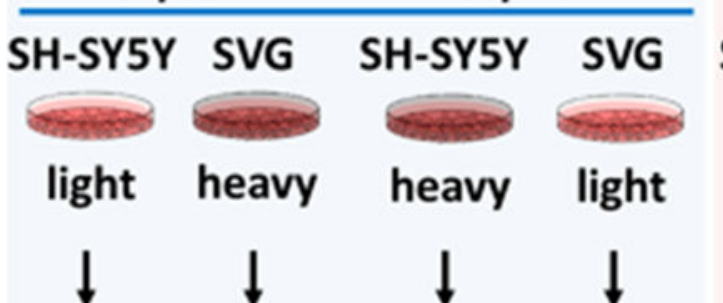

cell lysis, reduction, alkylation
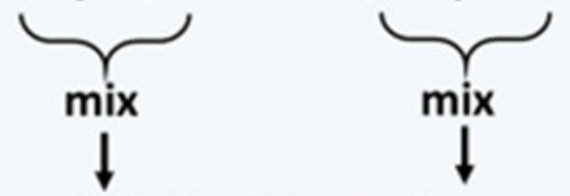

CH3-MeOH PPT, digest

with Lys $\mathrm{C}$ and then trypsin

Figure 1.

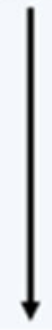

Basic $\mathrm{pH}$ reversed-phase

chromatography fractionation (12 fractions)

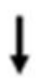

rep. 1

mTMT

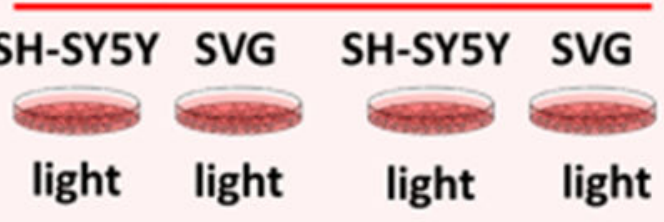<smiles>[131IH]</smiles>

cell lysis, reduction, alkylation

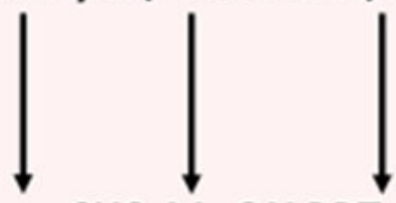

CH3-MeOH PPT, digest with LysC and then trypsin

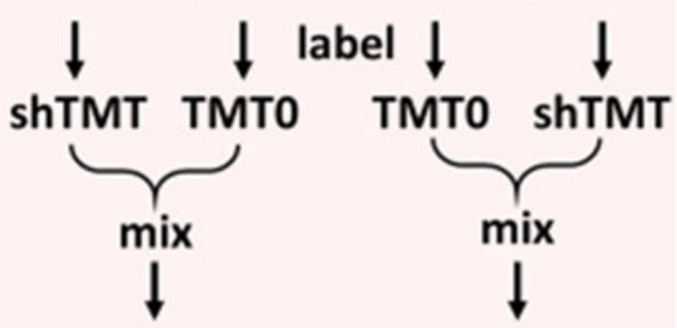

\section{MS2 analysis on an Orbitrap Fusion Lumos mass spectrometer}

mTMT structures and workflow overview. (A) Structures of TMT0 (left) and shTMT (right).

The 11 heavy atoms are labeled on shTMT as red asterisks. (B) Workflow overview. SHSY5Y and SVGp12 cell lines were cultured in DMEM/10\% dialyzed FBS media. For SILAC experiments, cells were also labeled with Lys8 and Arg 10 in place of the light versions of these amino acids. SILAC-labeled cells were mixed prior to protein precipitation, whereas sample mixing for the mTMT experiment was performed further downstream. Chloroform-methanol precipitation $\left(\mathrm{CH}_{3}-\mathrm{MeOH}\right.$ PPT), enzymatic digestion 
(with LysC and trypsin), high-pH reversed-phase chromatography, and mass spectrometry analysis were performed identically in both workflows. The samples were fractionated into 24 fractions, 12 of which were analyzed on an Orbitrap Fusion Lumos mass spectrometer over a 150 min LC gradient. 


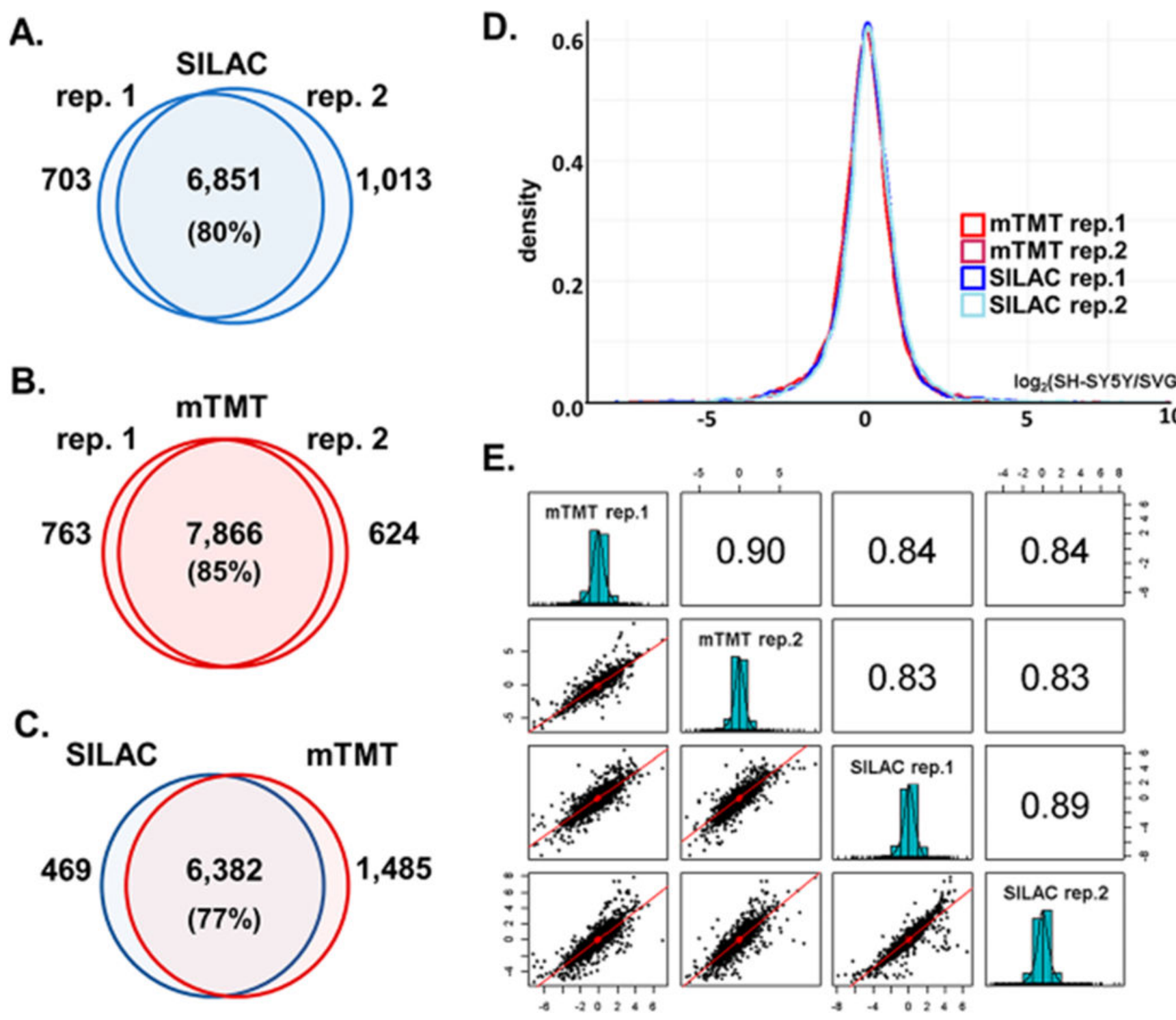

Figure 2.

SILAC and mTMT showing high correlation between reciprocal labeling and different workflows. (A-C) Venn diagrams comparing all quantified proteins between (A) SILAC replicates, (B) mTMT replicates, and (C) SILAC and mTMT workflows. (D) Overlay of smooth histograms of fold-changes for proteins common among all four data sets $(n=$ 6382). (E) Scatter plot matrix illustrating the correlation of the TMT relative abundance values for proteins common across all replicates of the SILAC and mTMT workflows ( $n=$ 6382). Values to the right of the diagonal are the Pearson correlation coefficients $(r)$ for each scatterplot shown to the left of the diagonal. 

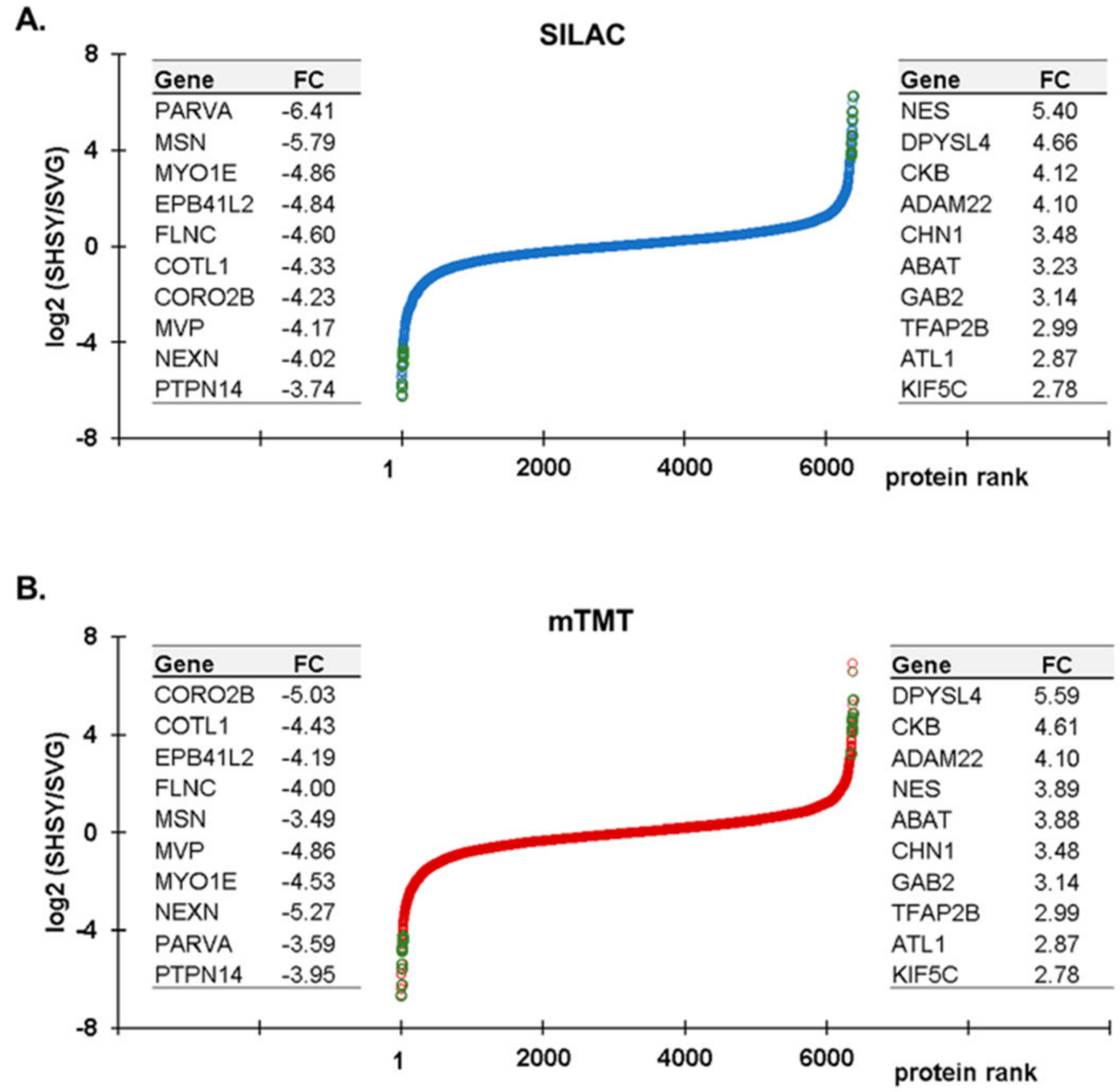

Figure 3.

Protein-level ranked fold-changes (FCs): line plots with proteins ranked according to increasing $\log _{2}$ ratios (i.e., the TMT relative abundance of SH-SY5Y to SVGp12) for (A) SILAC and (B) mTMT data sets. The values plotted are the $\log _{2}$ ratios of the average relative abundance value (SH-SY5Y/SVGp12) for each data set. On the tables to the left and right of the plot are proteins that are more highly abundant in SVGp12 or SH-SY5Y, respectively. The proteins listed are among the 50 most differentially abundant in both cell lines. $\mathrm{FC}=\log _{2}(\mathrm{SH}-\mathrm{SY} 5 \mathrm{Y} / \mathrm{SVGp} 12)$. 
Table 1.

\section{Data Overview}

\begin{tabular}{|c|c|c|c|c|c|c|}
\hline & \multirow[b]{2}{*}{ replicate } & \multirow{2}{*}{$\begin{array}{c}\text { peptides } \\
\text { unique }\end{array}$} & \multicolumn{2}{|c|}{ proteins } & \multicolumn{2}{|c|}{ differentially abundant $^{a}$} \\
\hline & & & identified & quantified $^{b}$ & up & down \\
\hline \multirow[t]{2}{*}{ mTMT } & 1 & 65885 & 9370 & 8629 & 118 & 218 \\
\hline & 2 & 63028 & 9225 & 8490 & & \\
\hline \multirow[t]{2}{*}{ SILAC } & 1 & 58334 & 9141 & 7553 & 136 & 208 \\
\hline & 2 & 63054 & 9475 & 7863 & & \\
\hline
\end{tabular}

${ }^{a}$ Differentially abundant refers to proteins that demonstrate a $\log _{2}$ ratio exceeding \pm 2 between cell lines.

${ }^{b}$ Quantified proteins have measurements in both heavy and light peptides. 